EGU21-4642

https://doi.org/10.5194/egusphere-egu21-4642

EGU General Assembly 2021

(c) Author(s) 2021. This work is distributed under

the Creative Commons Attribution 4.0 License.

\title{
Semi-structured mass movement interactions with flow-blocking mitigation
}

\author{
Bastian van den Bout ${ }^{1}$, Cees van Westen ${ }^{2}$, Victor Jetten ${ }^{3}$, and Om Dhakal ${ }^{4}$ \\ ${ }^{1}$ Twente University, ITC, Earth System Analysis, Apeldoorn, Netherlands (b.vandenbout@utwente.nl) \\ ${ }^{2}$ Twente University, ITC, Earth System Analysis, Apeldoorn, Netherlands (c.j.vanwesten@utwente.nl) \\ ${ }^{3}$ Twente University, ITC, Earth System Analysis, Apeldoorn, Netherlands (v.g.jetten@utwente.nl) \\ ${ }^{4}$ Twente University, ITC, Earth System Analysis, Apeldoorn, Netherlands (o.p.dhakal@students.utwente.nl)
}

The development of a failure plane in the subsurface can result in movement of large volumes of solids. Depending on the type of rock and initiation mechanism, this material can partially contain cohesive structure. Such semi-structured landslides or rock avalanches feature alternate dynamics compared to debris flows and other types of granular movements. The way in which this structure influences the impact on structures, and thus the resulting hazard, remains largely unknown. Recently, a two-phase semi-structured generalized mass movement model was developed. This model implements a full stress-strain relationship for the moving mixture. In this work, this model is applied to a set of hypothetical and real mitigation measures and structures. The effect of various types of checkdams and blocking pillars is investigated. The resulting impacts are converted to impact pressures and potential damage fractions. Finally, the model results are compared with traditional unstructured debris flow and landslide runout models. Results indicate the strong increase of structural damage with increased cohesive structure. The model furthermore predicts complex interactions between the semi-structured mass movements and mitigation measures. Moving aggregates can break, after which individual rocks or particles might continue in diverging trajectories. Depending on the physical parameters of the material, fragmentation can also occur before impact, after which movement is similar to granular flows. 